\title{
Routine hospital management of self-harm and risk of further self-harm: propensity score analysis using record-based cohort data
}

\author{
S. Steeg ${ }^{1 *}$, R. Emsley ${ }^{2}$, M. Carr ${ }^{1}$, J. Cooper ${ }^{1}$ and N. Kapur ${ }^{1,3}$ \\ ${ }^{1}$ Division of Psychology and Mental Health, Centre for Suicide Prevention, University of Manchester, Manchester Academic Health Science Centre, \\ Manchester, UK \\ ${ }^{2}$ Centre for Biostatistics, School of Health Sciences, University of Manchester, Manchester Academic Health Science Centre, Manchester, UK \\ ${ }^{3}$ Greater Manchester Mental Health NHS Foundation Trust, Manchester, UK
}

Background. The care received by people presenting to hospital following self-harm varies and it is unclear how different types of treatment affect risk of further self-harm.

\begin{abstract}
Method. Observational cohort data from the Manchester Self-Harm Project, UK, included 16456 individuals presenting to an Emergency Department with self-harm between 2003 and 2011. Individuals were followed up for 12 months. We also used data from a smaller cohort of individuals presenting to 31 hospitals in England during a 3-month period in 2010/2011, followed up for 6 months. Propensity score (PS) methods were used to address observed confounding. Missing data were imputed using multiple imputation.

Results. Following PS stratification, those who received a psychosocial assessment had a lower risk of repeat hospital attendance for self-harm than those who were not assessed [RR 0.87, 95\% confidence interval (CI) 0.80-0.95]. The risk was reduced most among people less likely to be assessed. Following PS matching, we found no associations between risks of repeat self-harm and admission to a medical bed, referral to outpatient psychiatry or admission to a psychiatric bed. We did not find a relationship between psychosocial assessment and repeat self-harm in the 31 centre cohort.

Conclusions. This study shows the potential value of using novel statistical techniques in large mental health datasets to estimate treatment effects. We found that specialist psychosocial assessment may reduce the risk of repeat self-harm. This type of routine care should be provided for all individuals who present to hospital after self-harm, regardless of perceived risk.
\end{abstract}

Received 17 September 2016; Revised 20 April 2017; Accepted 25 May 2017; First published online 22 June 2017

Key words: Outcomes, propensity score, psychosocial assessment, self-harm, treatment.

\section{Introduction}

People who intentionally self-poison, self-cut or otherwise injure themselves, acts referred to collectively as 'self-harm', have increased risks of suicide (Hawton et al. 2015), premature mortality (Bergen et al. 2012) and further acts of self-harm (Kapur et al. 2006). Guidance states that all people presenting to the ED after self-harm should receive a psychosocial assessment by a mental health specialist (NICE, 2011). In practice, however, just over half of individuals in England were found to receive a specialist psychosocial assessment (Cooper et al. 2013). Furthermore, this was found to vary between $22 \%$ and $88 \%$ in 31 hospitals in 2010-2011 (Cooper et al. 2013). Other types of hospital management following self-harm

\footnotetext{
* Address for correspondence: Sarah Steeg, Centre for Suicide Prevention, 2nd Floor Jean McFarlane Building, University of Manchester, Oxford Road, Manchester M13 9PL, UK.

(Email: sarah.steeg@manchester.ac.uk)
}

would not be suitable aftercare for every individual and allocation is based on clinical need (e.g., psychiatric inpatient admission is reserved for those with the greatest need). Nationally, there appears to be weak consensus about the approach to the assessment and management of individuals who present with selfharm, with levels of referral to outpatient care found to be between $11 \%$ and $64 \%$ and psychiatric admission between $0 \%$ and $21 \%$ in 31 English hospitals (Cooper et al. 2013; Quinlivan et al. 2014).

Studies examining effectiveness of psychological treatments that address self-harm are relatively numerous (Hawton et al. 2016), but few have examined standard care and how this might affect outcome. Routine aspects of care are not well suited to experimental approaches such as randomised controlled trials (RCTs). This is partly due to difficulties in obtaining a 'control group'; withholding treatment to selected individuals is not feasible in a real-world setting. These problems are further compounded within selfharm research settings due to the potential severe

This is an Open Access article, distributed under the terms of the Creative Commons Attribution licence (http://creativecommons.org/licenses/by/4.0/), which permits unrestricted re-use, distribution, and reproduction in any medium, provided the original work is properly cited. 
consequences of non-treatment, such as increased risks of further self-harm and suicide (Hickey et al. 2001).

Despite these difficulties, RCTs have provided some valuable information about effects of routine interventions following self-harm. An enhanced, 'therapeutic assessment' provided to adolescents following selfharm was not found to reduce self-harm compared with the usual assessment, though was associated with improved engagement with treatment (Ougrin et al. 2013). Approaches using telephone and postal contact following self-harm have been studied in RCTs; results have been mixed though some studies have reported a reduction in the number of repeat selfharm episodes (Milner et al. 2015). An RCT of general hospital admission following self-harm did not find any difference in future risk of self-harm by treatment group (Waterhouse \& Platt, 1990). However, these types of studies are carried out with small samples, and the 'routine' nature of the care received is likely to be compromised by the research conditions.

Alternative approaches, such as observing cohorts of patients over time to monitor future events, have provided useful information about the possible links between hospital care and outcomes. For example, by adjusting for observed confounders, assessment appeared to reduce the risk of repeat self-harm in some hospitals but not in others (Kapur et al. 2013). The use of instrumental variable analysis, a more complex statistical technique to address confounding, resulted in a similar finding (Carroll et al. 2016). In another study, assessment was associated with increased risk of suicide even after adjusting for observed confounders, suggesting there were unobserved differences between the groups (Kapur et al. 2015). A systematic review and meta-analysis of 64 studies found that the proportion of patients receiving an assessment in hospital was not associated with the rate of repeat self-harm (Carroll et al. 2014). However, this study also noted variation in the level of information about confounding factors such as previous self-harm.

When comparing outcomes, missing data and the lack of a 'control' group are commonly encountered challenges when evaluating effects of treatments using observational data. Methods used to handle missing data include listwise deletion, simple imputation and multiple imputation (Sterne et al. 2009). Approaches to address confounding by indication within health research include regression with multivariable adjustment, propensity score (PS) methods and the use of instrumental variables. PS matching (Erlangsen et al. 2014) and instrumental variable technique (Carroll et al. 2016) have recently been used to examine possible effects of interventions in cohorts of individuals who have self-harmed.
PS methods allow measured characteristics between individuals allocated to different treatments to be balanced. The PS, estimated for each participant in a study, represents the probability of treatment assignment given a set of measured covariates (Rosenbaum \& Rubin, 1983). There are a number of advantages of PS approaches over covariate adjustment methods. PS methods enable explicit comparison of treatment groups, and allow readers to judge how any remaining differences might affect the interpretation of the effect estimates. PS methods also allow comparisons to be made within restricted populations, such as treated and untreated patients within a pre-specified distance in their PSs.

The aim of the present study was to estimate associations between hospital management of self-harm and risks of repeat self-harm. In order to estimate treatment effects, we set out to use PS methods to adjust for differences in baseline characteristics between individuals receiving different treatments.

\section{Methods}

The STROBE guidelines for reporting observational cohort studies were followed (von Elm et al. 2008).

\section{Data sources}

The primary data source used for this study was a cohort containing information from hospital records for individuals presenting to Emergency Department (ED) after self-harm. Basic demographic and clinical information (including age, gender, ethnic group, employment status, marital status, place of residence, date and time of presentation and the method of harm) was extracted from the ED records by administrative research staff. In addition, information was collected from a proforma specifically designed for ED clinicians assessing patients presenting with self-harm (Bickley et al. 2013). The information in these proformas corresponded exactly to the variables in the database so was inputted directly. For those who were subsequently referred for a specialist mental health assessment, information was also extracted from the assessment notes. Research staff coded the information contained within the clinical assessment to the study variables. Researchers followed a protocol when coding assessments and when there was uncertainty about the coding the researchers discussed the anonymised case as a team. Inter-rater reliability was maximised by a period of training for all researchers where a random selection of assessments were coded separately then compared. This helped to identify areas of inconsistency and clarify coding rules. Validation exercises of the proformas against clinical records have 
showed excellent levels of agreement $(\kappa \geqslant 0.8$ for individual variables) (Kapur et al. 2006). Individuals attending one of the study hospitals with self-harm more than once were linked to identify repeat episodes. Most individuals were linked using National Health Service number, and where this was not available a combination of name, date of birth, postcode and ED number was used. The study was granted Section 251 approval by the National Information Governance Board (now the Confidential Advisory Group and the Health Research Authority) for the use of patient data.

The cohort is the result of a city-wide study covering hospitals in the city of Manchester, UK. The city has a population of around 520000 (Office for National Statistics, 2014) and is relatively deprived (Department for Communities and Local Government, 2008). Data were most comprehensive from 2003 onwards so we selected this as the start date. From this date, 10 years of data were available (2003-2012). For this study we included over 35 variables relating to the history of the individual, their mental state at the time of the self-harm and circumstances surrounding the act (Bickley et al. 2013).

The Manchester cohort represents a single city. To examine possible generalisability we conducted a secondary analysis on a separate, similar cohort of individuals presenting to hospital after self-harm. This cohort contained less detailed data from 31 centres across England (Cooper et al. 2013). The exact time periods differed, but data collection for each site took place within a 3-month period during 2010 and 2011. The same methodological approach used in the primary analysis, detailed below, was used for this secondary analysis.

\section{Categories of hospital management}

Four types of hospital management were examined: specialist psychosocial assessment, admission to a medical bed, referral to outpatient psychiatric services and psychiatric admission. Specialist psychosocial assessment consists of comprehensive assessment of the social and psychological circumstances of individuals' self-harm (NICE, 2011). The assessment also includes the formulation of a management plan for follow-up care, based on needs and risks considered during the assessment. A mental health specialist, usually a mental health nurse, psychiatrist or social worker, carries out the assessment which typically lasts around $1 \mathrm{~h}$ (NICE, 2011). Medical admission can include admission to a hospital ward or a short-stay observation or assessment unit, which now exist in most hospitals (Cooper et al. 2013). Referral to outpatient psychiatric services can include referral back to existing care or a new referral. Outpatient settings include crisis resolution home treatment teams, community mental health teams, community drug and alcohol teams, psychological therapy and referral to a psychiatrist. Psychiatric admission refers to either voluntary or involuntary (i.e. following a mental health act assessment) admission to a bed on a psychiatric ward. These four management approaches were not mutually exclusive so individuals in a comparison group for one treatment may have received one of the other treatments and those in the treated group may have also received additional care.

\section{Outcome measures}

Each individual's first presentation within the study period was used as the 'index' episode. A repeat presentation to any of the study hospitals within 12 months following the index episode was the primary outcome. Each individual was followed up for a minimum of 12 months from the date of their index episode to allow repeat presentations to be monitored.

\section{Missing data imputation}

Patterns of missing data were examined and the following pragmatic approach was used. Depending on the characteristics of the missing data, one, or a combination of approaches was used: (i) listwise deletion, (ii) single imputation and (iii) multiple imputation.

(i) For variables with high levels of completeness (ranging from $>94.0 \%$ to $99.9 \%$ ), which included exposure and outcome variables, cases with any missing data were excluded from the analysis (Fig. 1).

(ii) For episodes where no assessment took place, data for certain variables were obtained from ED clinical records, which were more likely to have mentioned the characteristic if it was present in the individual than if it was not present. For example, the ED records might mention if a suicide note was left, but be less likely to mention the absence of a suicide note. This would have resulted in bias towards positive responses had the data been imputed based only on observed distributions. Therefore, missing values were imputed as negative (if there was no assessment) before multiple imputation was performed. This approach was used for the following variables: circumstances of the act (premeditation, attempt to avoid discovery, suicide note and suicidal intent) and symptoms of depression (feeling depressed, appearing depressed, sleep disturbance, appetite changes, feelings of hopelessness, suicidal thoughts, suicidal plans and hallucinations). 


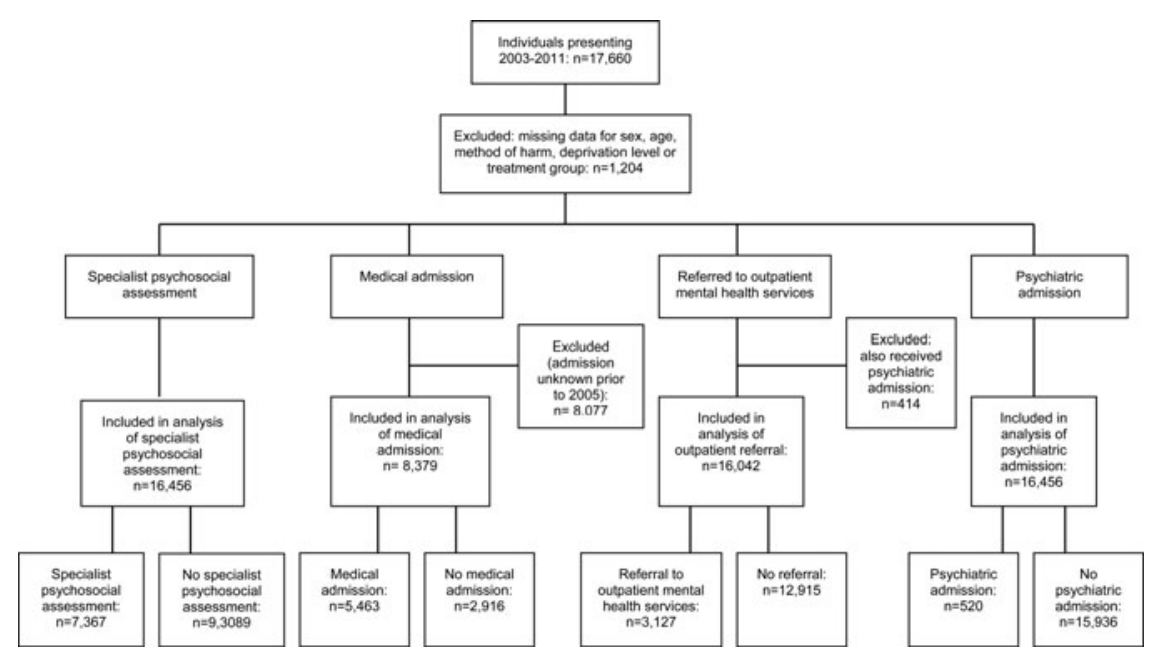

Fig. 1. Flow chart for individuals in the single-centre cohort.

(iii) Multiple imputation was using the 'chained equations' approach (Royston \& White, 2011) within Stata (Version 13.1) to generate ten imputed datasets. Multiple imputation was performed on the following variables regardless of assessment status: previous self-harm, current psychiatric treatment, previous psychiatric treatment, ethnic group, use of alcohol at the time of self-harm, alcohol use disorder, substance misuse, living situation, employment, and problems precipitating the self-harm (see Table 1 for the full list). The proportions of missing data ranged from $13 \%$ to $45 \%$ for imputed variables. The following data were used as additional predictors in the imputation model: sex, age, area-level deprivation, method of harm, repeat self-harm and treatment. The primary analysis was conducted on the imputed datasets. For comparison, analyses were repeated following listwise deletion of missing data, as recommended (Klebanoff \& Cole, 2008).

\section{Statistical analyses}

Risk ratios (RRs) for 12-month repeat self-harm were calculated using log-binomial regression models. First, unadjusted models were used to estimate the $R R$ of individuals receiving the treatment to those not receiving treatment. The comparison groups were individuals not receiving the treatment, regardless of other treatment that may have been received. One exception was for referral to outpatient psychiatry; individuals who were admitted to a psychiatric bed were excluded from the comparison group because they would have been likely to go on to receive outpatient care or an equivalent level of enhanced aftercare.
The PS was then estimated separately for each of the four categories of hospital management. A PS is a number between zero and one that is estimated for each individual. The score represents the probability of being allocated to a particular treatment, based on an individual's observed characteristics (Rosenbaum \& Rubin, 1983). This approach enables comparisons to be made between individuals with similar observed characteristics but who receive different treatments. Thirty five variables relating to the individual's clinical history, their social and demographic circumstances, mental state, problems leading up to the self-harm and details of the self-harm act were used to estimate the PS (see Table 1 for full list). The PSs were estimated by including these variables in a logistic regression model with treatment assignment as the dependent variable. The fit of the models was tested using a Hosmer-Lemeshow test (Lunt, 2014) and, where possible, interactions were included to improve the fit of the models. The PS was estimated separately in each of the ten imputed datasets and the average score was used as the PS (Mitra \& Reiter, 2012). The covariate balance between treated and untreated individuals before and after PS adjustment was compared. Additionally, balance in each PS quintile was examined. The region of common support was examined for each PS to see if different values of the PS were represented by both treated and untreated individuals.

Where there are treated and untreated individuals across the range of PSs, it is appropriate to estimate an average treatment effect (ATE) for the study population. In these instances the RR estimates were stratified by PS quintile to estimate the ATE. Estimates were also obtained within each strata of the PS.

For treatments that do not have treated and untreated subjects in all PS strata, it is more appropriate to estimate 
Table 1. Baseline v. PS stratified covariate balance of PS factors by psychosocial assessment, single-centre cohort $\left(N=16456^{\mathrm{a}}\right)$

\begin{tabular}{|c|c|c|c|c|c|c|}
\hline Subgroup & $\begin{array}{l}\text { Baseline } \\
\text { untreated, \% }\end{array}$ & $\begin{array}{l}\text { Baseline } \\
\text { treated, \% }\end{array}$ & $\begin{array}{l}\text { Standardised } \\
\text { difference }^{\mathrm{b}}\end{array}$ & $\begin{array}{l}\text { Stratified } \\
\text { untreated, \% }\end{array}$ & $\begin{array}{l}\text { Stratified } \\
\text { treated, \% }\end{array}$ & $\begin{array}{l}\text { Standardised } \\
\text { difference }\end{array}$ \\
\hline Total & $55.2(9089)$ & 44.8 (7367) & & & & \\
\hline Male & 44.0 & 41.3 & & 41.0 & 41.3 & \\
\hline Female & 56.0 & 58.7 & 0.05 & 59.0 & 57.7 & -0.01 \\
\hline Age 16-24 & 37.9 & 36.8 & -0.02 & 35.9 & 36.8 & 0.02 \\
\hline Age $25-44$ & 45.0 & 44.6 & -0.01 & 47.0 & 44.6 & -0.05 \\
\hline Age 45-64 & 13.8 & 17.2 & 0.10 & 14.0 & 17.2 & 0.09 \\
\hline Age 65+ & 3.3 & 1.4 & -0.13 & 3.1 & 1.4 & -0.12 \\
\hline White & 84.4 & 86.3 & 0.06 & 87.3 & 86.3 & -0.03 \\
\hline Black & 3.9 & 3.7 & -0.02 & 3.9 & 3.7 & -0.01 \\
\hline South Asian & 6.5 & 6.1 & -0.02 & 4.9 & 6.1 & 0.05 \\
\hline Other ethnic group & 5.1 & 4.0 & -0.06 & 4.0 & 4.0 & -0.001 \\
\hline Self-poison & 77.5 & 85.1 & 0.20 & 83.2 & 85.1 & 0.05 \\
\hline Self-cut & 16.2 & 10.4 & -0.17 & 12.8 & 10.4 & -0.07 \\
\hline Other self-injury & 6.3 & 4.4 & -0.09 & 4.0 & 4.4 & 0.02 \\
\hline Alcohol taken & 53.8 & 56.6 & 0.06 & 56.1 & 56.6 & 0.01 \\
\hline \multicolumn{7}{|l|}{ Receipt of psychiatric treatment } \\
\hline Current & 29.0 & 44.1 & 0.32 & 45.9 & 44.1 & -0.04 \\
\hline In the past 12 months & 25.7 & 23.0 & -0.06 & 35.7 & 23.0 & -0.28 \\
\hline More than 12 months ago & 12.1 & 23.0 & 0.29 & 17.4 & 23.0 & 0.14 \\
\hline Any previous self-harm & 41.1 & 57.1 & 0.32 & 55.9 & 57.1 & 0.02 \\
\hline Self-harm premeditated & 10.3 & 15.8 & 0.22 & 19.5 & 15.8 & -0.10 \\
\hline Avoided discovery & 5.1 & 10.9 & 0.22 & 12.5 & 10.9 & -0.05 \\
\hline Suicide note & 2.9 & 12.4 & 0.36 & 11.7 & 12.4 & 0.02 \\
\hline Wanted to die & 19.6 & 59.3 & 0.89 & 61.9 & 59.3 & -0.05 \\
\hline Current alcohol misuse & 28.0 & 32.1 & 0.09 & 33.4 & 32.1 & -0.03 \\
\hline Current substance misuse & 10.7 & 18.4 & 0.22 & 16.8 & 18.4 & 0.04 \\
\hline Feels depressed & 30.1 & 57.4 & 0.57 & 68.3 & 57.4 & -0.23 \\
\hline Looks depressed & 22.0 & 40.5 & 0.41 & 49.3 & 40.5 & -0.18 \\
\hline Sleep disturbance & 21.8 & 58.6 & 0.81 & 65.2 & 58.6 & -0.14 \\
\hline Appetite disturbance & 17.2 & 45.1 & 0.63 & 50.3 & 45.1 & -0.10 \\
\hline Hopelessness & 19.8 & 26.9 & 0.17 & 36.6 & 26.9 & -0.21 \\
\hline Suicidal thoughts & 18.5 & 30.0 & 0.27 & 38.5 & 30.0 & -0.18 \\
\hline Suicidal plans & 8.6 & 8.5 & -0.008 & 13.1 & 8.2 & -0.16 \\
\hline Hallucinations & 3.1 & 6.7 & 0.17 & 7.8 & 6.7 & -0.04 \\
\hline Homeless or living in a hostel & 4.4 & 4.5 & 0.007 & 5.0 & 4.5 & -0.02 \\
\hline Lives alone & 18.0 & 20.3 & 0.06 & 22.1 & 20.3 & -0.04 \\
\hline $\begin{array}{l}\text { Lives with family (partner/parents/ } \\
\text { siblings/children) }\end{array}$ & 63.6 & 61.6 & -0.04 & 57.8 & 61.6 & 0.08 \\
\hline Lives with friends/other relatives & 9.5 & 10.2 & 0.02 & 10.6 & 10.2 & -0.01 \\
\hline $\begin{array}{l}\text { Lives in supported or other } \\
\text { accommodation }\end{array}$ & 4.5 & 3.3 & -0.06 & 4.5 & 3.3 & -0.06 \\
\hline Employed & 30.5 & 33.3 & 0.06 & 29.9 & 33.3 & 0.07 \\
\hline Unemployed & 41.1 & 34.8 & -0.13 & 40.9 & 34.8 & -0.13 \\
\hline Registered sick & 3.9 & 9.9 & 0.24 & 7.4 & 9.9 & 0.09 \\
\hline Student & 14.2 & 13.5 & -0.02 & 11.9 & 13.5 & 0.05 \\
\hline Other ${ }^{c}$ & 10.2 & 8.6 & -0.06 & 9.9 & 8.6 & -0.05 \\
\hline \multicolumn{7}{|l|}{ Problems precipitating self-harm } \\
\hline Relationship with partner & 50.1 & 46.0 & -0.08 & 46.6 & 46.0 & -0.01 \\
\hline Relationship with family & 18.0 & 18.9 & 0.02 & 19.8 & 18.9 & -0.02 \\
\hline Relationship with others & 10.9 & 14.1 & 0.11 & 14.2 & 14.1 & 0.004 \\
\hline Bullying & 3.4 & 6.1 & 0.13 & 6.0 & 6.1 & 0.01 \\
\hline Bereavement & 6.8 & 11.3 & 0.16 & 11.4 & 11.3 & -0.001 \\
\hline Housing & 6.0 & 11.8 & 0.20 & 11.3 & 11.8 & 0.02 \\
\hline Work/study & 10.6 & 16.2 & 0.17 & 16.0 & 16.2 & 0.01 \\
\hline
\end{tabular}


Table 1 (cont.)

\begin{tabular}{|c|c|c|c|c|c|c|}
\hline Subgroup & $\begin{array}{l}\text { Baseline } \\
\text { untreated, \% }\end{array}$ & $\begin{array}{l}\text { Baseline } \\
\text { treated, \% }\end{array}$ & $\begin{array}{l}\text { Standardised } \\
\text { difference }^{\mathrm{b}}\end{array}$ & $\begin{array}{l}\text { Stratified } \\
\text { untreated, \% }\end{array}$ & $\begin{array}{l}\text { Stratified } \\
\text { treated, \% }\end{array}$ & $\begin{array}{l}\text { Standardised } \\
\text { difference }\end{array}$ \\
\hline Physical health & 6.3 & 9.5 & 0.12 & 9.1 & 9.5 & 0.01 \\
\hline Money & 6.9 & 13.9 & 0.23 & 13.2 & 13.9 & 0.02 \\
\hline Response to mental health symptoms & 4.9 & 14.9 & 0.34 & 14.3 & 14.9 & 0.02 \\
\hline Abuse & 2.8 & 7.6 & 0.21 & 6.9 & 7.6 & 0.03 \\
\hline Mean IMD score (high = deprived) & 44.5 & 43.6 & -0.04 & 43.0 & 43.6 & 0.03 \\
\hline
\end{tabular}

\footnotetext{
a Pooled proportions for multiply imputed data.

${ }^{b}$ The standard difference is the difference between the two proportions (treatment minus control) divided by the (mean) standard error of the two proportions.

' Other' includes housepersons, carers and retired.
}

the ATE on the treated (ATT). This estimate represents the treatment effect within the population that could feasibly receive the treatment. To obtain these estimates each treated individual was matched $1: 1$ to an untreated individual using nearest-neighbour Mahalanobis metric matching (D'Agostino, 1998). The Stata code used to undertake the matching is available on request from the corresponding author. A caliper of 0.25 of the standard deviation of the PS was imposed to restrict the distance between the matched pairs (Liu et al. 2013). Within this approach, replacement of controls was also allowed due to the restricted pool of controls within certain PS quintiles for some treatments. The number of times each untreated control could be matched was restricted to five to prevent the estimates reflecting a small number of controls used repeatedly (Stuart, 2010). The characteristics of the matched samples were compared to the full samples to see if they differed.

To test for differences in the effect estimates by subgroups, interaction terms for sex, age, previous self-harm, current and past psychiatric treatment, method of harm, ethnic group and area-based deprivation level were included in the adjusted and matched models.

\section{Sensitivity analysis}

The potential effects of an unobserved confounder were examined using a method proposed by VanderWeele and Arah (VanderWeele \& Arah, 2011; Liu et al. 2013) whereby we determined the necessary influence a confounder would need to have in order for the treatment effect to be no longer significant. We identified a potential unmeasured confounder from the existing literature as an illustrative example (Liu et al. 2013).

\section{Results}

\section{Description of cohorts}

The primary analysis was conducted on individuals who first presented between 1 January 2003 and 31
December 2011: 16456 individuals for psychosocial assessment and psychiatric admission and 16042 for outpatient referral (Fig. 1). Individuals were more likely to be female ( $56.6 \%$ v. $43.4 \%$ male) and most presentations involved self-poisoning (79.9\%). Data for medical admission were restricted to the later years in the study period (1 January 2005 to 31 December 2012) so analyses were carried out on a restricted sample of 8379. Individuals were followed up to the end of 2012 , resulting in a total number of 64878 personyears. 2476/16 456 (15.1\%) individuals presented with a further self-harm episode within 12 months.

\section{Pre and post PS stratification: balance of covariates between treatment groups}

There were substantial baseline differences between individuals receiving all four types of hospital management. For example, differences between the nonassessed and assessed groups were seen in the majority of variables (25/34 had a standardised difference of $>0.1$ ) (Table 1). Following stratification by PS quintile, most of these differences were balanced. However, people who received an assessment were more likely to have been in receipt of psychiatric treatment more than a year prior to the self-harm but less likely to have received more recent treatment. They were less likely to be presenting with symptoms of depression, to have planned the act and to be unemployed (Table 1). (See online Supplement for details of the other treatments.)

\section{Common support of PS by treatment group}

For psychosocial assessment, there was adequate overlap of treated and untreated in all propensity quintiles (online Supplementary Fig. S1). For individuals admitted to a medical bed, very few had PSs in the first two strata $(\mathrm{PS}<0.4)$. For outpatient referral, there were few untreated individuals in the upper two strata $(>0.6)$ 
and for psychiatric admission there were few untreated individuals with PSs $>0.2$. (These figures are available from the corresponding author on request.) Therefore, it was not possible to estimate a population ATE for these three treatments so the ATTs following PS matching were estimated instead.

\section{ATE estimates}

$44.8 \%$ of individuals received a psychosocial assessment. Unadjusted RRs suggested there was no association between psychosocial assessment and 12-month risk of repeat self-harm [RR 1.03, 95\% confidence interval (CI) 0.96 to 1.11]. After adjusting for PS strata, the RR reduced to 0.87 (CI 0.80-0.95), indicating a $13 \%$ reduction in risk of repeat self-harm for those receiving assessment. There were differences by propensity quintile; risk was significantly reduced in the second and third strata (strata 2: RR 0.75, CI 0.75 to 0.98; strata 3: 0.78, CI 0.67-0.92) but not in the first, fourth and fifth (Fig. 2). This indicates that those with lower likelihood of receiving an assessment benefit the most in terms of a reduced risk of further self-harm. There were some differences by patient subgroup: the magnitude of the risk reduction was greater for those with no previous self-harm (RR 0.81, CI 0.680.95, $p$ value for interaction 0.02 ) and those who used a method of harm other than poisoning or laceration (RR 0.64, CI 0.43-0.95, $p<0.001$ ). There did not appear to be marked differences in the magnitude of the effect estimate over time: the RR for 3-month repetition was 0.81 (CI 0.72-0.92) and was $0.84(0.76-0.93)$ for 6-month repetition.

\section{Average treatment effects on the treated (ATT)}

The majority of the 5463 individuals admitted to a medical bed were matched to a non-admitted control (5424). 62.9\% (3410) of the controls were used once, with the rest used between two and five times (Fig. 3). The only observed difference was the matched sample included a higher proportion of individuals who self-poisoned than the full cohort. Medical admission was not associated with any change in the risk of repeat self-harm in this sample: RR 1.10 (CI 0.98-1.24). This risk was lower for those with a history of selfharm (RR 1.02, CI 0.89-1.16, $p$ value for interaction = 0.01 ) and individuals already receiving psychiatric treatment (RR 0.99, CI 0.85-1.15, $p$ value 0.008).

Among the 3127 individuals referred for outpatient mental health treatment, 99\% (3109) were matched to an untreated pair. $48.5 \%$ (1507/3109) of the controls were used once. The matched sample included more individuals reporting specific problems leading up to the self-harm and depression symptoms, higher proportions of individuals in current psychiatric treatment and with a history of self-harm and more likely to have planned the act and have reported suicidal intent. In this sample, outpatient referral was not associated with a change in the risk of repeat self-harm: RR 1.10 (CI 0.97-1.23). However, there was an increased risk for those aged under 25 years (RR 1.29, CI 1.05-1.58, $p$ value for interaction $=0.01$ ).

In total 93.1\% (484/520) individuals receiving psychiatric admission were matched to a non-admitted control; and $78.7 \%(381 / 484)$ of the controls were used once. The profile of the matched sample was similar to that described above for the group referred to outpatient care, but with a greater magnitude of difference. The risk of repeat self-harm was similar between the matched pairs: RR 1.11 (CI 0.84-1.48). However, there was an increase for males (RR 1.72, CI 1.08-2.72, $p$ value for interaction 0.02).

\section{Comparison to listwise deletion}

In a sensitivity analysis, separate PSs were estimated for individuals in the cohort with complete data. The 12-month PS strata adjusted risks of repeat self-harm were, following assessment: RR 1.03 (CI 0.89-1.19); following medical admission: RR 1.10 (CI 0.87-1.37); outpatient mental health referral RR 1.17 (CI 1.01-1.34); psychiatric admission RR 1.13 (CI 0.85-1.50). However, there is likely to be substantial bias in these estimates due to the highly select nature of individuals in the sample that had complete data. Internal validity is also likely to be poor due to the lack of common support within propensity strata and poor covariate balance for the majority of variables (tables available upon request from the corresponding author).

\section{Sensitivity analyses to examine potential effect of unmeasured confounder}

It is estimated that up to $50 \%$ of $\mathrm{SH}$ patients have existing depression (Hawton et al. 2013) but this information is not always available from routine hospital data. A binary unobserved confounder with a prevalence of $45 \%$ in the untreated group and $53 \%$ in the treated group, which increased likelihood of treatment by $50 \%$, would need to increase in risk of repeat selfharm by $40 \%$ for the results to be no longer significant.

\section{Comparison to 31-site cohort}

For the 31-site study, 3465 (57.9\%) received a specialist psychosocial assessment, 3415/6153 (55.5\%) were admitted to a medical bed, $1727 / 5745$ (30.1\%) were referred for outpatient mental health care and 425/ $5865(7.2 \%)$ were admitted to a psychiatric bed. The 


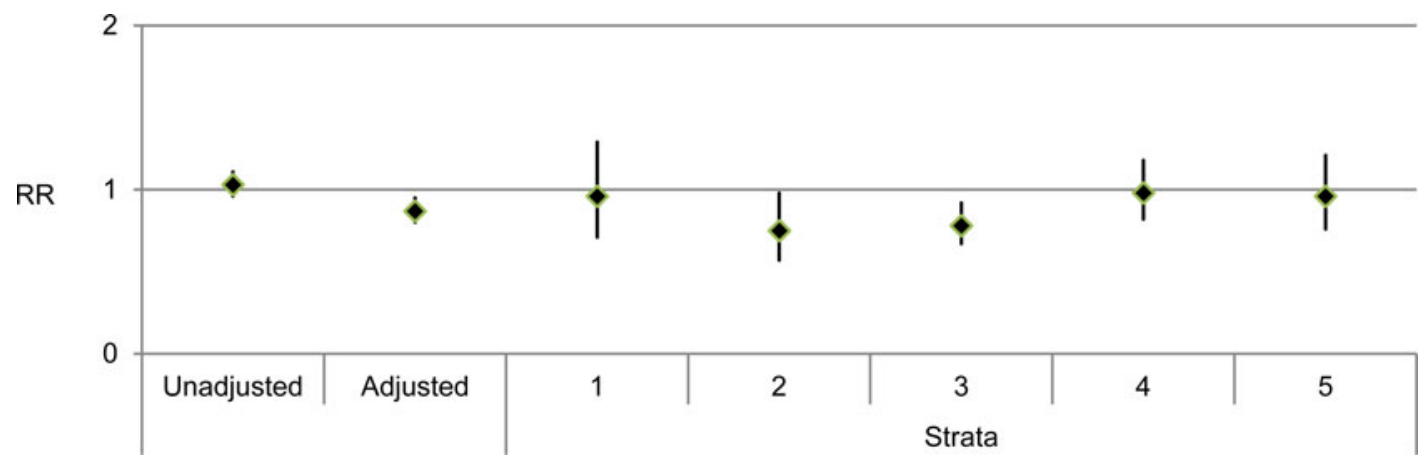

Fig. 2. Psychosocial assessment: Unadjusted, propensity score-adjusted, stratum-specific RRs.

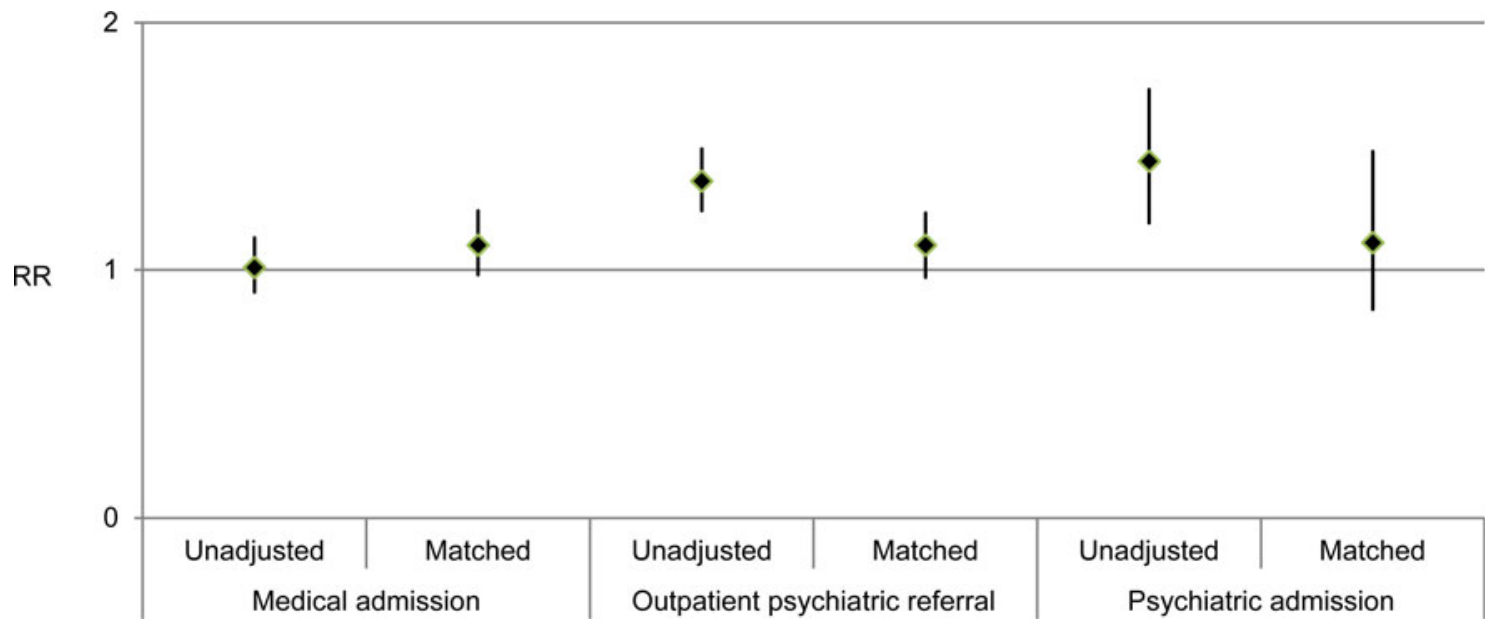

Fig. 3. Medical admission, outpatient psychiatric referral and psychiatric admission: unadjusted and propensity score-matched RRs.

follow-up period was a minimum of 6 months and $1236(20.7 \%)$ repeated within 6 months.

The PS strata adjusted 6-month risk of repeat selfharm following assessment was similar to the risk following no assessment in the 31-site cohort: RR 1.00 (CI 0.88-1.13). For the PS matched pairs there was an increased risk following medical admission: RR 1.14 (CI 1.03-1.25) but no difference in risk following psychiatric outpatient referral: RR 0.99 (CI 0.89-1.11) or psychiatric admission: RR 0.83 (CI 0.67-1.02).

\section{Discussion}

\section{Main findings}

The PSs successfully balanced the majority of the observed differences between treatment groups. Before the use of PS methods, no associations between psychosocial assessment or medical admission and the risk of repeat self-harm were found. Increased risks of repeat self-harm following referral to outpatient psychiatric treatment and admission to a psychiatric hospital were found, which probably reflected higher risk individuals receiving these forms of management (confounding by indication).

Following the use of PS methods, specialist psychosocial assessment appeared to reduce the risk of repeat self-harm. This association was not found in the 31-centre study. There did not appear to be any changes in risk of repeat self-harm following the other types of hospital management.

Specialist psychosocial assessment appeared to be more effective at reducing risk of repetition amongst those who were presenting with their first ever episode of self-harm, those who used a method of self-harm other than self-poisoning or cutting (including asphyxiation, jumping from a height and burning) and those who were in the lower PS strata (i.e. those who were less likely to receive assessment).

Medical admission was associated with a greater increased risk of repeat self-harm for individuals presenting with their first episode of self-harm and those with no existing psychiatric treatment. While there were no groups for which a referral to outpatient 
mental health services was associated with reduced risk of future self-harm, the estimated risk was higher for people aged under 25 . The estimated RR for selfharm following psychiatric inpatient admission was lower for females than males, with an increased risk seen for males.

\section{Strengths and limitations}

There are a number of ways to use PSs. Stratification by the PS produces an estimate of the ATE. The representative nature of the data is preserved, as participants are not excluded from the analysis. When the PS is used to match each treated subject to an untreated subject (or more than one) the ATT is estimated. This means that the comparison group is restricted to untreated individuals who are similar, in terms of what we know about them, to treated individuals. The treatments we were interested in vary from being intended/suitable everyone (e.g. specialist psychosocial assessment) to being reserved for those with the greatest clinical need (psychiatric inpatient admission). Where there are untreated subjects outside of the PS range of the treated, it is not appropriate to estimate the ATE (Stuart, 2010). Whilst we were interested in the effect of the clinical management on our self-harm population, this proved only to be possible for specialist psychosocial assessment.

Each method relies on the data containing information that adequately represents differences between treatment groups (measured confounders), as well as realistic and frank discussion of the extent to which this is likely. The estimated effect of a $13 \%$ reduction in the risk of repeat self-harm following specialist psychosocial assessment was small. The introduction of the potential 'unmeasured' confounder of depression demonstrated that, although small, the effect of specialist psychosocial assessment withstood a moderate unmeasured confounding effect.

Multiple imputation of missing data has previously been used when investigating outcomes following selfharm with large cohorts (Mars et al. 2014). While multiple imputation can increase precision and reduce bias, it is suggested that complete case as well as imputed results are presented for comparison (Klebanoff \& Cole, 2008). When data were restricted to the cohort with complete data, there was poor common support in PS strata (i.e. individuals with similar estimated likelihood of treatment could not be found in both treatment groups). There was also residual imbalance in covariates following stratification on the PS. This suggests hidden confounding is present; estimated treatment effects in these instances would therefore be biased and should be interpreted cautiously.
Data were more likely to be missing for individuals who did not receive specialist psychosocial assessment. While some factors influencing likelihood of assessment are practical (time of attendance, staff availability), others are driven by clinical judgement. We attempted to address this by accounting for assessment status when imputing missing data. Our specific approach to the single imputation of variables where there was a positive response bias required an assumption to be made that if the characteristic was not recorded for nonassessed individuals then it was not present. This is a strong assumption. This conservative approach to imputing values may have underestimated the factors associated with risk in these individuals. We postulate that this would underestimate the treatment effect estimate as a result of incomplete adjustment for these confounders in the PS model. However, the relatively large proportions of missing data for some of the variables should be considered when interpreting the estimates from the imputed data.

The estimates obtained from the 31-site multicentre cohort were similar on the whole to the results from the primary analyses, although the reduced risk following specialist psychosocial assessment was not observed. This difference may reflect the depth of detail available in the multicentre data. It is usually necessary to balance scale and detail; our results suggest cohort studies of self-harm are most useful for estimating effects of treatment when comprehensive data relating to predictors of treatment and factors related to risk of further self-harm are extracted from the clinical records. A previous multicentre study (Kapur et al. 2013) included data from the present study, though at a more basic level. While assessment was found to be associated with reduced risk of further self-harm in other centres, it was not the case for Manchester. The use of single-centre data in the present study enabled the use of more detailed information, such as the presence or absence of symptoms of depression, suicide note, planning of the act, avoidance of discovery and suicidal intent. One interpretation is that these are important confounding factors affecting clinical decisions regarding treatment and risk of further self-harm. However, the use of single-centre data limits the generalisability of findings.

The main limitation was the non-randomised allocation of the interventions. However we were interested in how the routine management of self-harm, outside of research settings, relates to individuals' future risk of self-harm. PS methods offer a robust and transparent approach to help address observed differences between treatment groups. Despite this, the balancing of unmeasured differences that would result from randomisation cannot be replicated using PS methods. Therefore, there is no guarantee that the treatment 
effect estimates are free from confounding. However, the observational nature of the study was also a strength. The level of case ascertainment was high, due to the method of data collection and individuals would be unlikely to attend a hospital outside of the study area for repeat presentations because the catchment area is relatively well-defined. This was a large study enabling subgroups to be examined.

A further limitation is that we were not able to follow up individuals to see if they actually attended their recommended treatment such as outpatient appointments and primary care. There are also factors outside of the immediate clinical management to consider, such as availability, quality and extent of outpatient and primary care once the patient leaves the ED. Furthermore, due to the intensive nature of the data collection for large scale observational studies such as this, the setting does not necessarily reflect present-day hospital services. Changes, such as the introduction of the RAID service, which now has increased responsibility for assessing individuals who self-harm, may have resulted in higher rates of assessment and follow-up care.

\section{Clinical and research implications}

Our findings suggest assessment is beneficial, not just to ensure appropriate treatment and follow-up for individuals, but also in reducing the burden of illness for individuals and easing pressure on hospitals. However, this finding was not supported by the 31-site study. While the effect in the single-centre study was at the populationlevel, a stronger reduction was seen for people who were presenting with their first episode of self-harm. While recommending assessment might in some senses be 'stating the obvious', it is also an important message to frontline clinical staff that simple interventions (a basic assessment) may help in a group of patients who some staff find it difficult to engage with. The proportion of individuals receiving assessment in English hospitals varies widely, suggesting some hospitals select individuals based on level of clinical severity. Where hospitals reserve assessment for higher risk presentations, our results suggest this is a missed opportunity to prevent repeat episodes of self-harm. A study carried out in Japan also reported that psychiatric intervention (consisting of assessment and/or psychotherapy) following hospital admission for drug overdose was associated with lower likelihood of readmission (Kanehara et al. 2015).

Medical admission was associated with an increased risk of further self-harm for people who had not previously self-harmed and those who were not receiving treatment for a mental health problem. This result could reflect the adverse effect of not receiving some kind of ongoing treatment at the time of self-harm despite a current crisis.
For people aged under 25, an increase in risk was observed for those referred to outpatient mental health services. It is possible that young people respond less well to the services offered to them than older people. A recent systematic review suggested psychological interventions might be effective for adults in reducing the number repeating self-harm (Hawton et al. 2016). The contrast with our findings could be due to the heterogeneity of follow-up care received by individuals studied here, compared to those participating in RCTs.

Psychiatric inpatient admission is an intensive intervention and is not suitable aftercare for most people who have self-harmed. An increase in risk following psychiatric admission was observed for males. This does not necessarily suggest that psychiatric admission is harmful, as has been suggested elsewhere (Large \& Ryan, 2014), but may reflect differences in male helpseeking behaviour following admission. The result may also be due to residual unmeasured confounding. A previous study reported possible associations between psychiatric admission following self-harm and reduced risk of premature mortality for some groups, including adults aged 65 years and over and people with a history of self-harm (Kapur et al. 2015).

Future research could focus on how individuals referred to outpatient mental health services following self-harm navigate their ongoing care. Qualitative research could provide insight into which components of treatment are helpful and perhaps highlight obstacles to attending follow-up care, particularly in younger age groups. Observational record-based data could help identify the routes taken by people after leaving hospital and identify vulnerable groups.

\section{Conclusions}

Understanding more about how routine care provided to self-harm patients relates to future risk is vital for services and patients. A specialist psychosocial assessment is an opportunity to identify treatment needs and plan follow-up care, while referrals to psychiatric outpatient or inpatient treatment can provide longer-term care. The assessment itself may also be helpful. Specialist psychosocial assessment appeared to reduce the risk of subsequent repeat self-harm, but this finding was limited to the single-centre study. This effect was observed at the population level, strengthening the evidence for the provision of assessment for all individuals, regardless of perceived risk.

\section{Supplementary Material}

The supplementary material for this article can be found at https://doi.org/10.1017/S0033291717001702. 


\section{Acknowledgements}

This work was supported by funding from an National Institute for Health Research (NIHR) Doctoral Research Fellowship (DRF 2013-06-070). The paper also uses data collected as part of independent research funded by the NIHR under its Programme Grants for Applied Research Programme (Grant Reference Number RP-PG-0606-1247) (Principal Investigators: Professors David Gunnell, Keith Hawton and Nav Kapur). The views expressed are those of the authors and not necessarily those of the NHS, the National Institute for Health Research or the Department of Health. We would like to thank the study team at the Manchester Self-Harm Project, University of Manchester, for collecting the data.

\section{Declaration of interest}

NK chaired the NICE self-harm guideline and the NICE depression in adults guideline.

\section{References}

Bergen H, Hawton K, Waters K, Ness J, Cooper J, Steeg S, Kapur N (2012). Premature death after self-harm: a multicentre cohort study. Lancet 380, 1568-1574.

Bickley H, Steeg S, Turnbull P, Haigh M, Donaldson I, Matthews V, Dickson S, Kapur N, Cooper J (2013). Self-Harm in Manchester: January 2010 to December 2011. Manchester Self-Harm Project: Manchester, UK.

Carroll R, Metcalfe C, Gunnell D (2014). Hospital management of self-harm patients and risk of repetition: systematic review and meta-analysis. Journal of Affective Disorders 168, 476-483.

Carroll R, Metcalfe C, Steeg S, Davies NM, Cooper J, Kapur N, Gunnell D (2016). Psychosocial assessment of self-harm patients and risk of repeat presentation: an instrumental variable analysis using time of hospital presentation. PLoS ONE 11, e0149713.

Cooper J, Steeg S, Bennewith O, Lowe M, Gunnell D, House A, Hawton K, Kapur N (2013). Are hospital services for self-harm getting better? An observational study examining management, service provision and temporal trends in England. BMJ Open 3, e003444.

D'Agostino RB (1998). Propensity score methods for bias reduction in the comparison of a treatment to a non-randomized control group. Statistics in Medicine 17, 2265-2281.

Department for Communities and Local Government (2008). The English Indices of Deprivation 2007 (http://www.gov. uk/government/collections/english-indices-of-deprivation).

Erlangsen A, Lind BD, Stuart EA, Qin P, Stenager E, Larsen KJ, Wang AG, Hvid M, Nielsen AC, Pedersen CM, Winsløv J, Langhoff C, Mühlmann C, Nordentoft M (2014). Short-term and long-term effects of psychosocial therapy for people after deliberate self-harm: a register-based, nationwide multicentre study using propensity score matching. Lancet Psychiatry 2, 49-58.

Hawton K, Bergen HCooper J, Turnbull P, Waters K, Ness J, Kapur N (2015). Suicide following self-harm: findings from the multicentre study of self-harm in England, 2000-2012. Journal of Affective Disorders 175, 147-151.

Hawton K, Saunders K, Topiwala A, Haw C (2013). Psychiatric disorders in patients presenting to hospital following self-harm: A systematic review. Journal of Affective Disorders 151, 821-830.

Hawton K, Witt KG, Salisbury TLT, Arensman E, Gunnell D, Hazell P, Townsend E, van Heeringen K (2016). Psychosocial interventions for self-harm in adults. Cochrane Database of Systematic Reviews, Issue 5, CD012189.

Hickey L, Hawton K, Fagg J, Weitzel H (2001). Deliberate self-harm patients who leave the accident and emergency department without a psychiatric assessment - a neglected population at risk of suicide. Journal of Psychosomatic Research 50, 87-93.

Kanehara A, Yamana H, Yasunaga H, Matsui H, Ando S, Okamura T, Kumakura Y, Kiyohide Fushimi K, Kasai K (2015). Psychiatric intervention and repeated admission to emergency centres due to drug overdose. BJPsych Open 1, 158-163.

Kapur N, Cooper J, King-Hele S, Webb R, Lawlor M, Rodway C, Appleby L (2006). The repetition of suicidal behavior: a multicenter cohort study. Journal of Clinical Psychiatry 67, 1599-1609.

Kapur N, Steeg S, Turnbull P, Webb R, Bergen H, Hawton K, Geulayov G, Townsend E, Ness J, Waters K, Cooper J (2015). Hospital management of suicidal behaviour and subsequent mortality: a prospective cohort study. Lancet Psychiatry 2, 809-816.

Kapur N, Steeg S, Webb R, Haigh M, Bergen H, Hawton K, Ness J, Waters K, Cooper J (2013). Does clinical management improve outcomes following self-harm? Results from the multicentre study of self-harm in England. PLOS ONE 8, e70434.

Klebanoff MA, Cole SR (2008). Use of multiple imputation in the epidemiologic literature. American Journal of Epidemiology 168, 355-357.

Large MM, Ryan CJ (2014). Disturbing findings about the risk of suicide and psychiatric hospitals. Social Psychiatry and Psychiatric Epidemiology 49, 1353-1355.

Liu WW, Kuramoto SJ, Stuart EA (2013). An introduction to sensitivity analysis for unobserved confounding in nonexperimental prevention research. Prevention Science 14, 570-580.

Lunt M (2014). Propensity Analysis in Stata Revision: 1.1 (http://personalpages.manchester.ac.uk/staff/mark.lunt/ propensity_guide.pdf) University of Manchester.

Mars B, Heron J, Crane C, Hawton K, Lewis G, Macleod J, Tilling K, Gunnell D (2014). Clinical and social outcomes of adolescent self harm: population based birth cohort study. British Medical Journal 349, G5954.

Milner AJ, Carter G, Pirkis J, Robinson J, Spittal MJ (2015). Letters, green cards, telephone calls and postcards: systematic and meta-analytic review of brief contact 
interventions for reducing self-harm, suicide attempts and suicide. British Journal of Psychiatry 206, 184-190.

Mitra R, Reiter JP (2012). A comparison of two methods of estimating propensity scores after multiple imputation. Statistical Methods in Medical Research 25, 188-204.

NICE (2011). The Long Term Care and Treatment of Self-Harm. Clinical Guideline 133. National Institute of Health and Care Excellence: London, UK.

Office for National Statistics (2014). Neighbourhood Statistics. ONS: London, UK.

Ougrin D, Boege I, Stahl D, Banarsee R, Taylor E (2013). Randomised controlled trial of therapeutic assessment versus usual assessment in adolescents with self-harm: 2-year follow-up. Archives of Disease in Childhood 98, 772-776.

Quinlivan L, Cooper J, Steeg S, Davies L, Hawton K, Gunnell D, Kapur N (2014). Scales for predicting risk following self-harm: an observational study in 32 hospitals in England. BMJ open 4, e004732-e004732.

Rosenbaum PR, Rubin DB (1983). The central role of the propensity score in observational studies for causal effects. Biometrika 70, 41-55.
Royston P, White IR (2011). Multiple Imputation by Chained Equations (MICE): implementation in Stata. Journal of Statistical Software 45, 1-20.

Sterne JAC, White IR, Carlin JB, Spratt M, Royston P, Kenward MG, Wood AM, Carpenter JR (2009).

Multiple imputation for missing data in epidemiological and clinical research: potential and pitfalls. British Medical Journal 338, b2393.

Stuart EA (2010). Matching methods for causal inference: a review and a look forward. Statistical Science 25, 1-21.

VanderWeele TJ, Arah OA (2011). Bias formulas for sensitivity analysis of unmeasured confounding for general outcomes, treatments, and confounders. Epidemiology 22, $42-52$.

von Elm E, Altman DG, Egger M, Pocock SJ, Gotzsche PC, Vandenbroucke JP, Initiative S (2008). The Strengthening the Reporting of Observational Studies in Epidemiology (STROBE) statement: guidelines for reporting observational studies. Journal of Clinical Epidemiology 61, 344-349.

Waterhouse J, Platt S (1990). General-hospital admission in the management of parasuicide - a randomized controlled trial. British Journal of Psychiatry 156, 236-242. 